\title{
Cerebral Microvascular Accumulation of Tau Oligomers in Alzheimer's Disease and Related Tauopathies
}

\begin{abstract}
Diana L Castillo-Carranza ${ }^{1,2}$, Ashley N Nilson ${ }^{1,2}$, Candice E Van Skike ${ }^{4}$, Jordan B Jahrling ${ }^{4}$, Kishan Patel $^{1,2}$, Prajesh Garach ${ }^{1,2}$, Julia E Gerson ${ }^{1,2}$, Urmi Sengupta ${ }^{1,2}$, Jose Abisambra ${ }^{5}$, Peter Nelson $^{6}$, Juan Troncoso ${ }^{7}$, Zoltan Ungvari ${ }^{8}$, Veronica Galvan ${ }^{4}$, Rakez Kayed ${ }^{1,2,3, *}$

${ }^{1}$ Mitchell Center for Neurodegenerative Diseases, University of Texas Medical Branch, Galveston, TX 77555, USA; ${ }^{2}$ Departments of Neurology, Neuroscience and Cell Biology, University of Texas Medical Branch, Galveston, TX 77555, USA; ${ }^{3}$ Sealy Center for Vaccine Development, University of Texas Medical Branch, Galveston, TX 77555, USA; ${ }^{4}$ Department of Cellular and Integrative Physiology and The Barshop Institute for Longevity and Aging Studies, University of Texas Health Science Center at San Antonio, TX 78245, USA; ${ }^{5}$ Sanders-Brown Center on Aging and Department of Physiology, University of Kentucky, Lexington, KY 40536, USA; ${ }^{6}$ Division of Neuropathology and Sanders-Brown Center on Aging, University of Kentucky, Lexington, KY 40536, USA; ${ }^{7}$ Clinical and Neuropathology Core, Department of Medicine, Johns Hopkins University, Baltimore, MD 21287, USA; ${ }^{8}$ Department of Geriatric Medicine and Reynolds Oklahoma Center on Aging, University of Oklahoma Health Sciences Center, Oklahoma, OK 73104, USA
\end{abstract}

[Received December 15, 2016; Revised January 10, 2017; Accepted January 12, 2017]

\begin{abstract}
The importance of vascular contributions to cognitive impairment and dementia (VCID) associated with Alzheimer's disease (AD) and related neurodegenerative diseases is increasingly recognized, however, the underlying mechanisms remain obscure. There is growing evidence that in addition to A $\beta$ deposition, accumulation of hyperphosphorylated oligomeric tau contributes significantly to AD etiology. Tau oligomers are toxic and it has been suggested that they propagate in a "prion-like" fashion, inducing endogenous tau misfolding in cells. Their role in VCID, however, is not yet understood. The present study was designed to determine the severity of vascular deposition of oligomeric tau in the brain in patients with AD and related tauopathies, including dementia with Lewy bodies (DLB) and progressive supranuclear palsy (PSP). Further, we examined a potential link between vascular deposition of fibrillar A $\beta$ and that of tau oligomers in the Tg2576 mouse model. We found that tau oligomers accumulate in cerebral microvasculature of human patients with AD and PSP, in association with vascular endothelial and smooth muscle cells. Cerebrovascular deposition of tau oligomers was also found in DLB patients. We also show that tau oligomers accumulate in cerebral microvasculature of Tg2576 mice, partially in association with cerebrovascular A $\beta$ deposits. Thus, our findings add to the growing evidence for multifaceted microvascular involvement in the pathogenesis of AD and other neurodegenerative diseases. Accumulation of tau oligomers may represent a potential novel mechanism by which functional and structural integrity of the cerebral microvessels is compromised.
\end{abstract}

Key words: tau, oligomers, tauopathies, cerebrovascular dysfunction, brain vascular dysfunction, cerebrovasculature, Alzheimer's disease

There is growing evidence that a spectrum of vascular and microvascular pathologies (including neurovascular uncoupling, endothelial dysfunction, blood brain barrier disruption, cerebral microhemorrhages etc) contribute to initiation and/or progression of neurodegenerative diseases, including Alzheimer's disease (AD) [1-4]. To recognize this paradigm, shift the phrase "vascular contributions to cognitive impairment and dementia

*Correspondence should be addressed to: Rakez Kayed, PhD, 301 University Blvd., Medical Research Building, Room 10.138C, Galveston, Texas 77555-1045, USA. Email: rakayed@utmb.edu.

Copyright: ( ) 2017. This is an open-access article distributed under the terms of the Creative Commons Attribution License (CC BY 4.0), which permits unrestricted use, distribution, and reproduction in any medium, provided the original author and source are credited. 
(VCID)" was introduced [5-7]. The VCID concept provides mechanistic explanation to the well-established role of known vascular risk factors in exacerbation of $\mathrm{AD}$. Results from recent research suggest that VCID are manifested early during development of $\mathrm{AD}$ [8] and are amenable for therapeutic intervention to dementia.

The cellular and molecular mechanisms underlying VCID are not well understood. There are many studies extant that attribute cerebrovascular abnormalities in $\mathrm{AD}$ to the deposition of fibrillar $A \beta$ in the wall of cerebral vessels [9-12], a pathology referred to as cerebral amyloid angiopathy (CAA). However, there is increasing evidence that other molecular mechanisms also play an equally important role. Importantly, in addition to extracellular accumulation of $\mathrm{A} \beta$ the intracellular accumulation of the microtubule-associated protein tau is believed to contribute to the pathogenesis of neurodegeneration [13-15]. Under normal conditions tau binds to microtubules and assists with their formation and stabilization. In $\mathrm{AD}$ and other related neurodegenerative disorders (collectively called tauopathies) tau is hyperphosphorylated and misfolded, and accumulates into insoluble intracellular aggregates known as neurofibrillary tangles (NFT) [16, 17] which likely interfere with numerous cellular functions. In addition, recent research suggest that multimers comprised of two or more tau molecules, called tau oligomers [18] are a distinct toxic tau species and have the ability to translocate between neurons [19-21]. Tau oligomers thus exhibit "prion-like" propagation mechanisms and are capable of spreading in the brain by inducing endogenous tau phosphorylation and misfolding [22]. In addition to $\mathrm{AD}$, tau oligomers have been identified in other tauopathies including progressive supranuclear palsy (PSP) [23], Parkinson's disease (PD), dementia with Lewy bodies (DLB) [24] and in subjects with Huntington's disease (HD) [25] suggesting a common pathogenic role. Despite these advances, the role of oligomeric tau in cerebromicrovascular abnormalities associated with neurodegenerative diseases remains obscure.

The goal of the present study was to determine vascular deposition of oligomeric tau in the brain in patients with $\mathrm{AD}$ and other types of tauopathies, including DLB and PSP. Further, we examined a potential link between CAA and vascular deposition of tau oligomers in the Tg2576 mouse model.

\section{MATERIALS AND METHODS}

\section{Human samples}

Postmortem brain samples from AD, DLB, PSP, and agematched control subjects, were obtained from Oregon Health and Science University, the Institute for Brain
Aging and Dementia (University of California-Irvine, Irvine, California, USA) and the Brain Resource Center at Johns Hopkins.

\section{Animals}

All studies were performed under approval of the UTMB Institutional Animal Care and Use Committee [Animal Welfare Assurance Number D16-00202 (A3314-01)]. Tg2576 mice were bred at UTMB free of enrichment. Mice were housed at the UTMB animal care facility and maintained according to U.S. Department of Agriculture standards $(12 \mathrm{~h}$ light/dark cycle with food and water available ad libitum). Tg2576 mice overexpress amyloid precursor protein (APP) carrying the KM670/671NL ('Swedish') mutation and develop early amyloid plaques and abundant vascular amyloid deposition [26].. The brains of Tg2576 mice were examined at 3 and 23 months of age.

\section{Immunohistochemistry}

Immunohistochemistry was performed on paraffinembedded sections. Briefly, sections were deparaffinized using xylene and ethyl alcohol and subsequently rehydrated and washed three times in $1 \mathrm{X}$ phosphate buffered saline (PBS) for 5 minutes each. Non-specific antigens were blocked using normal goat serum at room temperature for 1 hour. Sections were incubated overnight with primary rabbit polyclonal anti-tau oligomer antibody (T22, 1:200). Sections were washed three times in PBS for $10 \mathrm{~min}$ and incubated with goat anti-rabbit IgG for 1 hour at room temperature. (Vectastin $\mathrm{ABC}$ kit; Vector Laboratories, Burlingame, CA). A 3, 3'-diaminobenzidine peroxidase substrate kit (Vector Laboratories) was used to visualize tau oligomer immunoreactivity. Hematoxylin was used to counterstain nuclei according to the manufacturer's instructions. Bright field images were acquired using a Nikon Eclipse 800 microscope equipped with a Nikon DXM1200 color CCD camera (Nikon Instruments, Inc., Melville, NY).

\section{Immunofluorescence}

Fluorescent immunohistochemistry was performed on paraffin-embedded sections. Briefly, paraffin sections were deparaffinized and rehydrated. Sections were blocked with goat serum for 1 hour and incubated overnight at $4{ }^{\circ} \mathrm{C}$ with rabbit anti-tau oligomer antibody T22 (1:200). The next day, sections were washed with PBS and incubated with goat anti-rabbit IgG Alexa-568 (1:350, Invitrogen) for 1 hour. The sections were then washed three times with PBS, followed by blocking in 5\% goat serum for 1 hour, then by overnight incubation with 
a smooth muscle actin antibody [SMA, 1:250 $(0.8 \mu \mathrm{g} / \mathrm{ml})$, Abcam], Lewy body antibody [LB509, 1:300 (3 $\mu \mathrm{g} / \mathrm{ml})$, Abcam], von Willebrand Factor antibody [vWF, 1:250 (4 $\mu \mathrm{g} / \mathrm{ml})$, Chemicon], $\mathrm{A} \beta$ antibody [6E10, 1:300 (3.33 $\mu \mathrm{g} / \mathrm{ml})$, Biolegend], or total tau antibody [Tau 5, 1:300 $(0.16 \mu \mathrm{g} / \mathrm{ml})$, Biolegend]. The next day, the sections were washed three times in PBS, followed by 1 hour incubation with goat anti-rabbit IgG or anti-mouse Alexa Fluor 488 (1:350; Invitrogen) depending on the species the primary antibody was raised in. The sections were then washed three times in PBS, stained with DAPI (Vector Laboratories) and mounted in Vectashield mounting media. The sections were examined using an epifluorescence microscope (Nikon Eclipse 800) equipped with a CoolSnap-FX monochrome CCD camera (Photometrics, Tucson, AZ, USA) using standard Nikon fluorescein isothiocyanate (FITC), Texas Red, and DAPI filters set for Alexa Fluor 488, Alexa Fluor 568 and DAPI respectively. Imaging and analysis were performed using Metavue V7.1 software (Molecular Devices), Adobe Photoshop and FIJI/Image J.
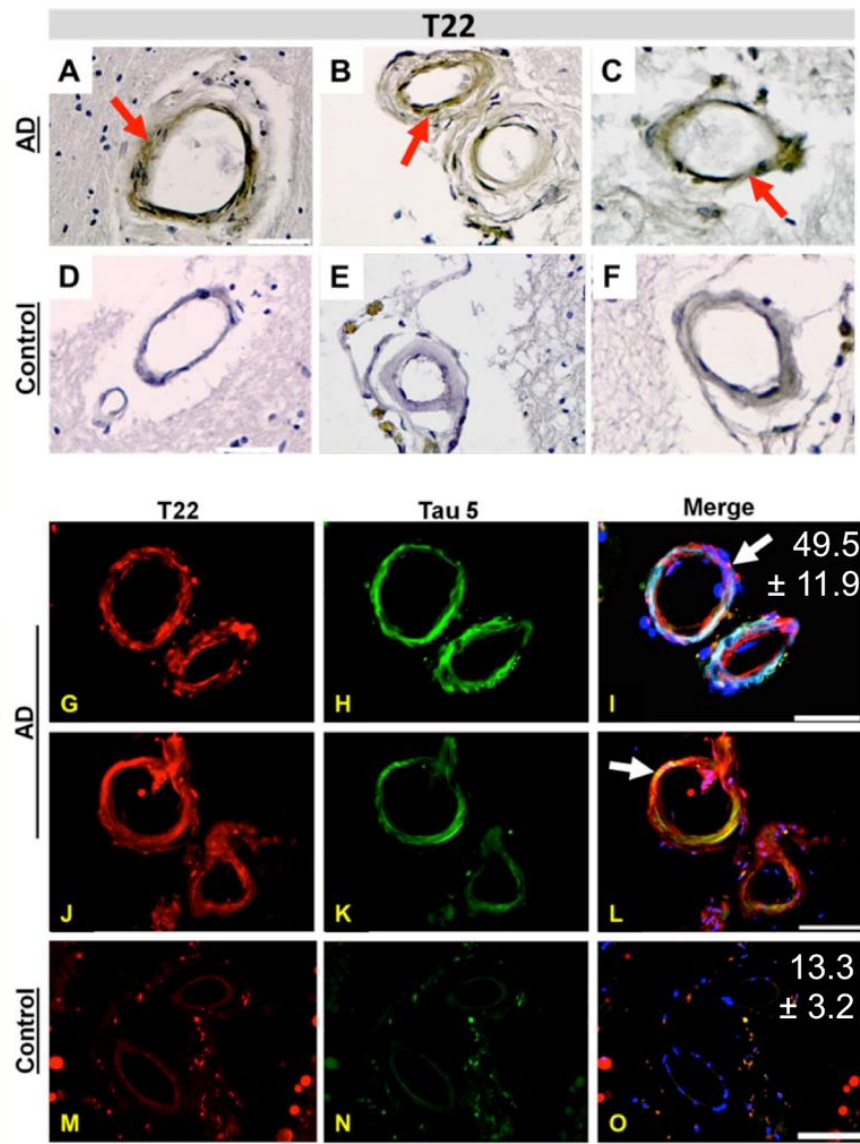

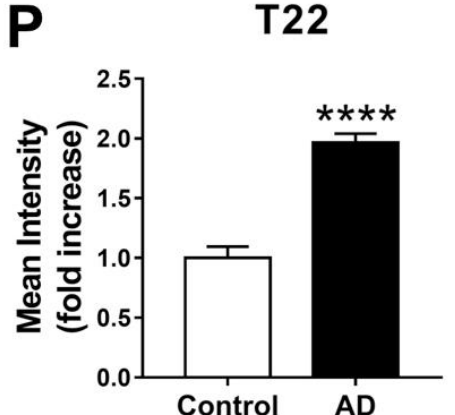

Q

Tau 5

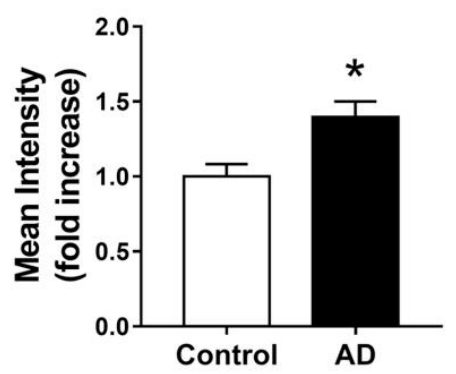

Figure 1. Deposition of tau oligomers in cerebrovasculature of human Alzheimer's Disease (AD) brains. (A-F) Representative images of immunohistochemistry studies using an oligomeric tau-specific antibody (T22) in cortical sections from $\mathrm{AD}(\mathrm{A}-\mathbf{C})$ and age-matched controls (D-F). (G-O) Representative images of oligomeric tau (T22, red), and total tau (tau 5, green) cerebrovascular immunoreactivity in cortical sections of AD (G-L) and age-matched control (M-O) brains. Quantitative analyses of mean fluorescence intensity show increased tau oligomer- (T22, P) and total tau-specific (Tau5, Q) immunoreactivity in vasculature of $\mathrm{AD}$ brains compared to age-matched controls [****, $\mathrm{t}(28)=8.12, p<0.0001$, and ${ }^{*}, \mathrm{t}(17)=2.39, p=0.029$, for T22 and tau5 immunoreactivity respectively]. Our tau oligomer antibody T22 [18, 41] has been validated by immunoblot, ELISA, coimmunoprecipitation as well as rodent and human tissue staining, is produced endotoxinfree, and is commercially available (Millipore ABN454). For all studies, $\mathrm{n}=3$ brains/group; 10-15 sections from each sample were analyzed for tau oligomers. All AD samples were tested and were positive for tau oligomers. Merged images are shown with DAPI (blue). In all panels, arrows indicate tau inclusions. Mean percent colocalization \pm SEM of T22 with Tau 5 is reported in the figure. Scale bar $50 \mu \mathrm{m}$. 


\section{Confocal imaging}

Images were collected using a Zeiss LSM-510 Meta confocal microscope with a $63 \times 1.20$ numerical aperture water immersion objective (UTMB Optical Microscopy Core). The images were obtained using three different excitation lines $(364,488$, and 543 ) by sequential acquisition. After excitation with 364-, 488- and 543lasers, line emissions were collected with $385-470-\mathrm{nm}$, 505-530-nm, and 560-615-nm filters, respectively. All images were collected using 8-frame-Kallman-averaging with a pixel time of $2.51 \mu \mathrm{s}$ and a pixel size of $160 \mathrm{~nm}$. Image processing and analysis were performed with Metamorph 7.2, LSM Image browser, and FIJI/Image J.

\section{Image analysis and statistics}

To obtain mean intensity measurements, mean gray values of immunofluorescent images were determined using the threshold technique in Image J. Briefly, original 8-bit grayscale images were uploaded, the threshold was adjusted to match the immunofluorescence contained in the image, single vascular elements were isolated with the selection tool, and the mean gray value of the thresholded area was measured and recorded. When applicable, Mander's colocalization coefficient was computed using the "coloc 2" plugin in the FIJI version of Image J. All differences between two group means were assessed with t-tests. When comparing 3 group means, a one-way ANOVA was used, followed by Tukey's posthoc.
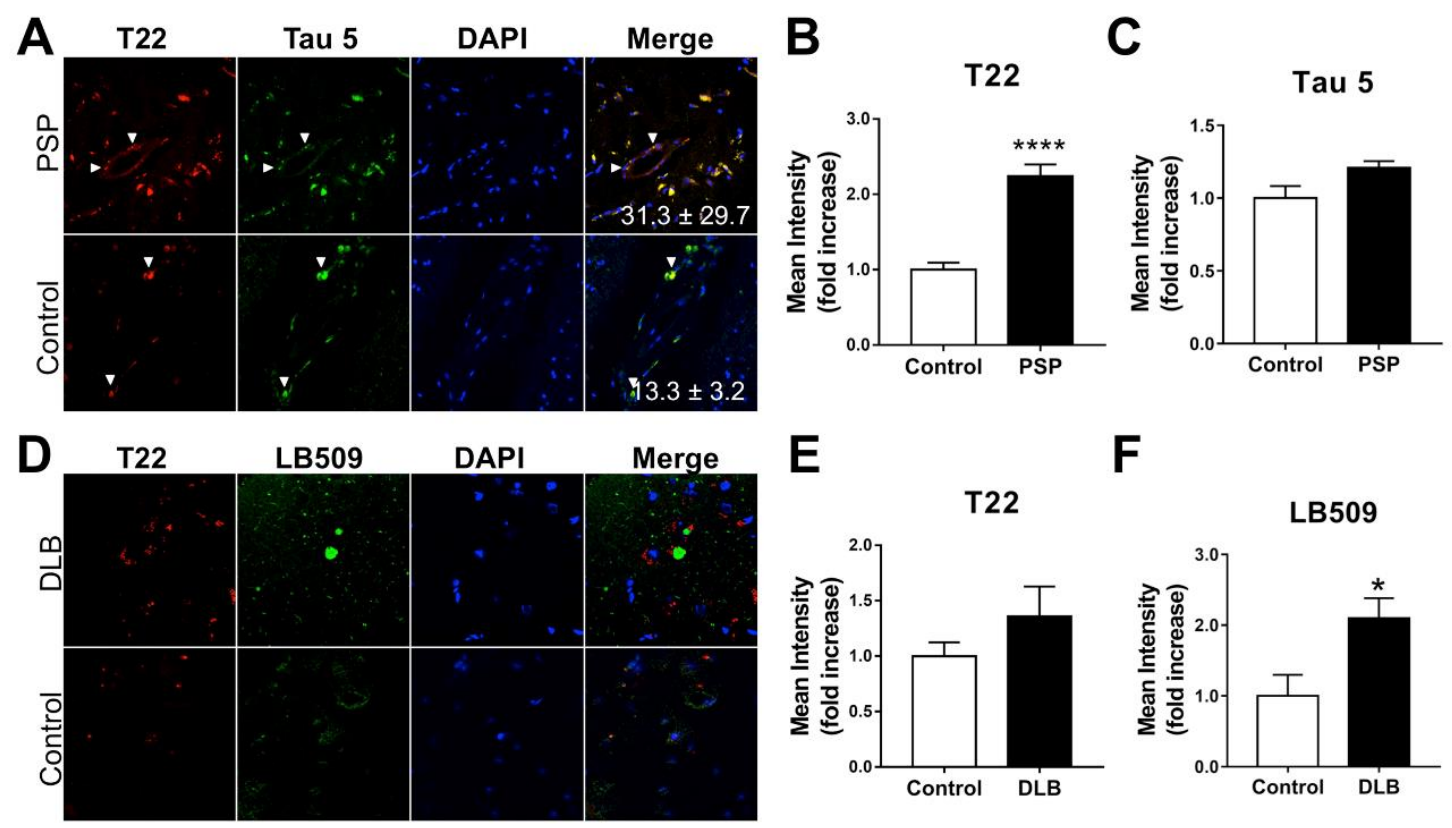

$\mathbf{F}$

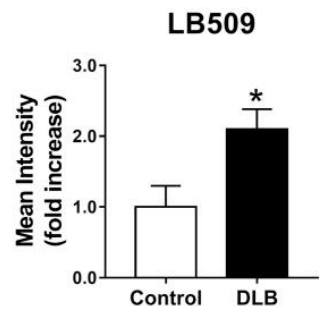

Figure 2. Increased deposition of tau oligomers in cerebrovasculature of patients with progressive supranuclear palsy (PSP) but not with dementia with Lewy bodies (DLB). (A) Representative images of pons sections from PSP patients and age-matched controls immunostained with antibodies specific for tau oligomers (T22, red) and total tau (Tau 5 , green). Quantitative analyses of mean fluorescent intensity shows (B) increased levels of tau oligomers [****, $\mathrm{t}(18)=$ 7.38, $p<0.0001]$ but not of total tau [C, $\mathrm{t}(7)=1.67, p=0.138]$ in cerebrovasculature of patients with PSP compared to age-matched controls. Examples of cerebrovascular oligomeric tau deposits are indicated with white arrows. (D) Representative images of brain sections from frontal cortex of DLB patients and age-matched controls immunostained with antibodies specific for tau oligomers (T22, red) and alpha-synuclein (LB509, green). (E) Quantitative analysis of mean fluorescence intensity did not reveal differences in oligomeric tau immunoreactivity in DLB patients compared to age-matched controls $(\mathrm{t}(9)=1.289, p=0.23)$. (F) Quantitative analysis of mean fluorescence intensity demonstrates an increase in alpha-synuclein abundance in brains of DLB patients compared to controls $(*, t(9)=2.486, p=0.035)$. For all studies, $\mathrm{n}=2$ brains/group; $10-15$ sections from each sample were analyzed for tau oligomers. All PSP and DLB samples were tested and were positive for tau oligomers. 


\section{RESULTS}

\section{Deposition of tau oligomers in AD brain vasculature}

Bright field images of both AD (Fig. 1A-C) and control (Fig. 1D-F) cortical brain sections stained for T22 revealed tau oligomers present within small blood vessel walls in AD brains; these deposits are notably absent in age-matched controls. To further investigate these findings, we utilized confocal microscopy to obtain fluorescent images of both oligomeric and total tau protein. Our findings indicate increased colocalization of tau oligomers with Tau 5 in AD brains (Fig. 1G-I and JL, with Mander's coefficients shown in white) compared to age-matched controls (Fig. 1M-O). Furthermore, the mean intensity of T22 was increased nearly $100 \%$ per vascular element in $\mathrm{AD}$ brains (Fig. 1P) while the mean intensity of Tau 5 was increased by nearly $50 \%$ in the same subjects (Fig. 1Q) when compared to controls. These data indicate that tau oligomers accumulate in $\mathrm{AD}$ brain vasculature.

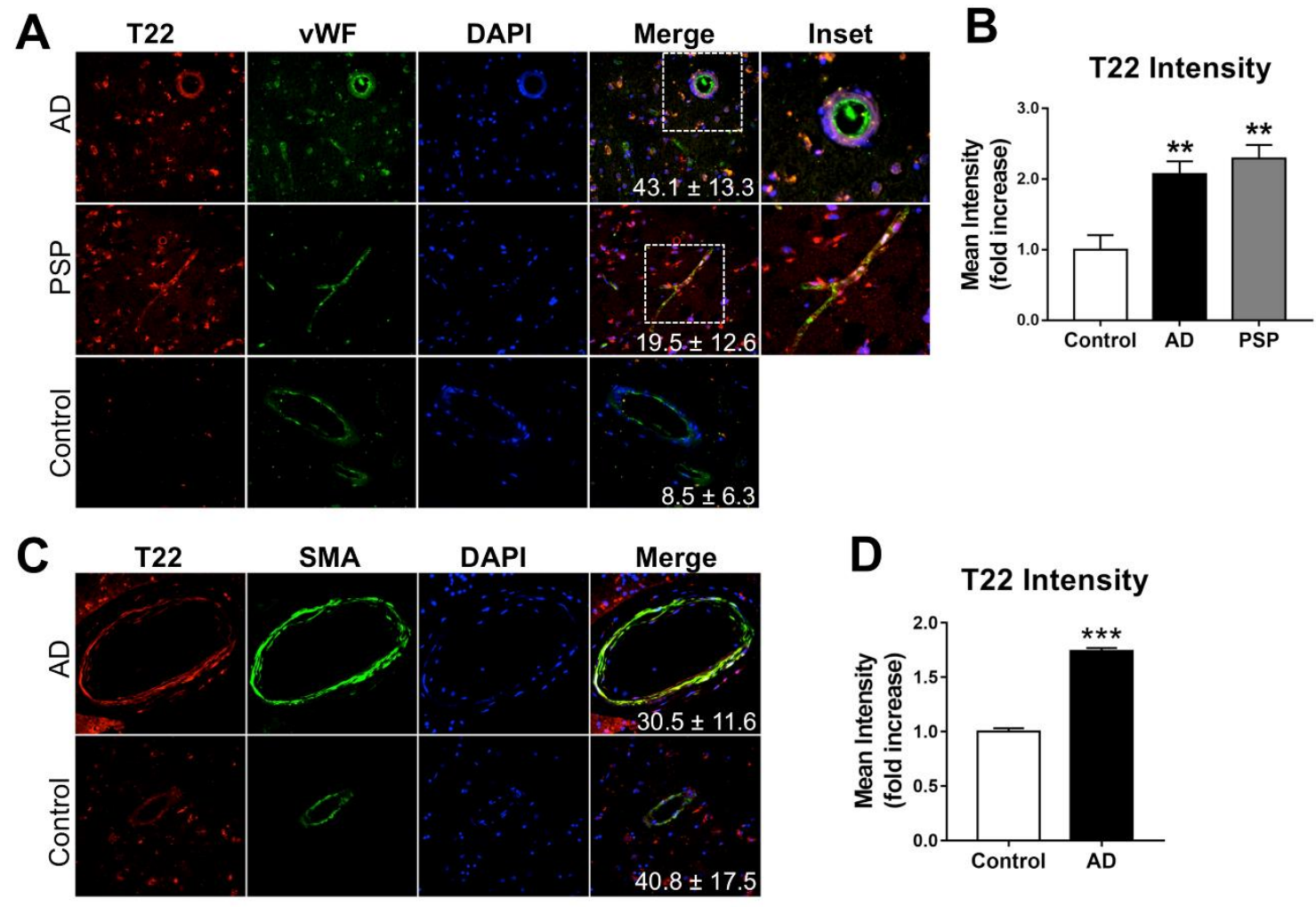

Figure 3. Association of tau oligomers with vascular endothelium and smooth muscle in Alzheimer's disease brain. Representative images of brain sections from AD patients and age-matched controls reacted with (A) antibodies specific for tau oligomers (T22, red) and the endothelial cell marker Von Willebrand Factor (vWF, green). (B) Quantitative analysis shows increased tau oligomer immuonoreactivity in the cerebrovasculature of AD and PSP patients compared to age-matched controls [Control-AD **, $\mathrm{q}=5.24$ and $p=0.0078$, Control-PSP $* *, \mathrm{q}=6.728$ and $\mathrm{p}=0.0013$, AD-PSP, $\mathrm{q}=1.03$ and $p=0.7517$ as a result of Tukey's post hoc test applied to a significant effect of group in ANOVA, $\mathrm{F}(2,12)=13.03$, p=0.001]. (C) Representative images of brain sections from AD patients and age-matched controls reacted with antibodies specific for tau oligomers (T22, red) and the vascular smooth muscle cell marker smooth muscle actin (SMA, green). (D) Quantitative analysis demonstrates increased tau oligomer immuonoreactivity associated with smooth muscle actin-positive cells $[* * *, \mathrm{t}(4)=15.35, p=0.0001)$. For all studies, $\mathrm{n}=2$ brains/group; 10-15 sections from each sample were analyzed for tau oligomers. All AD and PSP samples were tested and were positive for tau oligomers. Mean percent colocalization values \pm SEM are shown as insets. Data in panels B and D were also included in the analyses of tau oligomer abundance in Figure 1P. 


\section{Tau oligomers accumulate in cerebrovasculature of PSP and $D L B$ patients}

In order to determine whether vascular deposition of tau oligomers is common amongst tauopathies, we next determined tau oligomer deposition in the cerebrovasculature of PSP patients. Confocal images from the pons of PSP patients (Fig. 2A, upper panel) and age-matched control subjects (Fig. 2A, lower panel), were collected from sections immunostained using T22 and Tau 5 antibodies. Similar to our findings in AD subjects (Fig. 1), oligomeric tau immunoreactivity colocalized with Tau 5 immunoreactivity in vasculature of PSP brains and was largely absent in brains of age-matched control subjects. The mean intensity of oligomeric tau-specific immunoreactivity increased more than $100 \%$ in PSP subjects compared to age-matched controls (Fig. 2B), whereas a minimal and non-significant increase in total tau abundance was observed (Fig. 2C). These data indicate that, similar to our observations in $\mathrm{AD}$ brain (Fig. 1), tau oligomers preferentially accumulate in PSP cerebrovasculature.

We next determined localization and abundance of oligomeric tau and $\alpha$-synuclein in sections from frontal cortex of patients with DLB using immunohistochemistry with an $\alpha$-synuclein specific antibody (LB509) and T22. Our studies revealed deposition of tau oligomers in microvessel walls as well as in brain parenchyma of DLB brains (Fig. 2D, upper panel). Notably, Lewy body deposits were absent in microvasculature, but present as neuronal cytoplasmic deposits (arrow in Fig. 2D, upper panel) in the vicinity of blood vessels (Fig. 2D). Both oligomeric tau and $\alpha$-synuclein immunoreactivity were absent in control subjects (Fig. 2D, lower panel). Although a trend to increased oligomeric tau immunoreactivity was observed in brain microvasculature of DLB patients, this difference was not significant (Fig. $2 \mathrm{E})$. As expected, we observed a significant increase in $\alpha$ synuclein immunofluorescence in DLB subjects (Figure 2F).

\section{Tau oligomers are associated with endothelial cell markers in $A D$ and PSP}

We next sought to determine whether oligomeric tau associates with specific brain vascular cell types in $\mathrm{AD}$ brain. Cortical sections from AD as well as pons sections from PSP and age matched control brains were immunostained with T22 and with antibodies specific for the endothelial cell marker von Willebrand Factor (vWF). Tau oligomers were found in association with brain vascular endothelial cells in AD and PSP (Fig. 3A) and were virtually absent in control brains. Immunofluorescence intensity of T22 associated with the brain vascular endothelial cell compartment, however, was significantly increased in AD and PSP brains (Fig. 3B). These data are consistent with the observed overall increases in cerebrovascular oligomeric tau as well as total tau in AD (Fig. 1) and PSP (Fig. 2A-C) brains, and suggest that tau oligomers are found in association with brain vascular endothelial cells in various neurological disease states.

Smooth muscle cells are a critical component of brain artery and arteriole walls that participate in the regulation of blood flow [27]. We next sought to determine whether in addition to targeting vascular endothelial cells, tau oligomers associate with vascular smooth muscle cells in AD. To this aim we used confocal imaging on sections from $\mathrm{AD}$ patients and age-matched controls immunestained with T22 and with an antibody specific for smooth muscle actin (SMA), a marker of smooth muscle cells. Significant tau oligomer immunoreactivity was found in association with SMA immunoreactivity in arterioles in AD brain (Fig. 3C-D), suggesting that smooth muscle cells may also be a target of tau oligomer deposition. Together with our studies of Figure 1, these data demonstrate increased oligomeric tau in the cerebrovasculature of patients with $\mathrm{AD}$ compared to agematched controls (Fig. 3D).

\section{Tau oligomers and AB partially colocalize in cerebrovasculature of the Tg2576 mouse model of AD.}

A large body of evidence indicates that exposure to high levels of soluble forms of $A \beta[28,29]$ and fibrillar $A \beta$ deposition [30] are deleterious to cerebrovascular function [4]. Because we showed that oligomeric tau accumulates in cerebrovascular endothelial and smooth muscle cells in various tauopathies including $A D$, we next sought to determine whether vascular $A \beta$ and oligomeric tau may coexist in the cerebrovasculature. To this aim we used Tg2576 mice, which develop CAA-like cerebrovascular lesions starting at $\sim 11-12$ months of age that increase significantly with age, but are devoid of vascular fibrillar $A \beta$ at earlier ages [26, 31]. Tg2576 mice are largely presymptomatic at 3 months of age, as synaptic loss [32], alterations in neuronal plasticity, and decline in cognitive function do not arise until 4-5 months of age [33]. Oligomeric tau and $A \beta$ colocalization were measured in the cerebrovasculature of 3 and 23-monthold $\operatorname{Tg} 2576$ animals. We report abundant immunoreactivity for both tau oligomers and $A \beta$ in cerebrovasculature of Tg2576 animals at 23 months of age (Fig. 4A, upper panel), in contrast with absent cerebrovascular tau oligomer and $A \beta$ immunoreactivity in 3-month-old Tg2576 mice (Fig. 4A, lower panel). Notably, $28 \%$ of tau oligomers colocalize with $A \beta$ within the cerebrovasculature of 23-month-old Tg2576 mice 
(Fig. 4A, inset). As expected, we demonstrated an age dependent increase in oligomeric tau immunofluorescence (Fig. 4B) and A $\beta$ immunoreactivity (Fig. 4C) in 23-month-old Tg2576 mice compared to 3month-old mice. It is conceivable that $\mathrm{A} \beta$ and oligomeric tau may functionally and potentially physically interact in $\mathrm{AD}$ cerebrovasculature, and may cooperatively act to compromise brain vascular function in $\mathrm{AD}$.

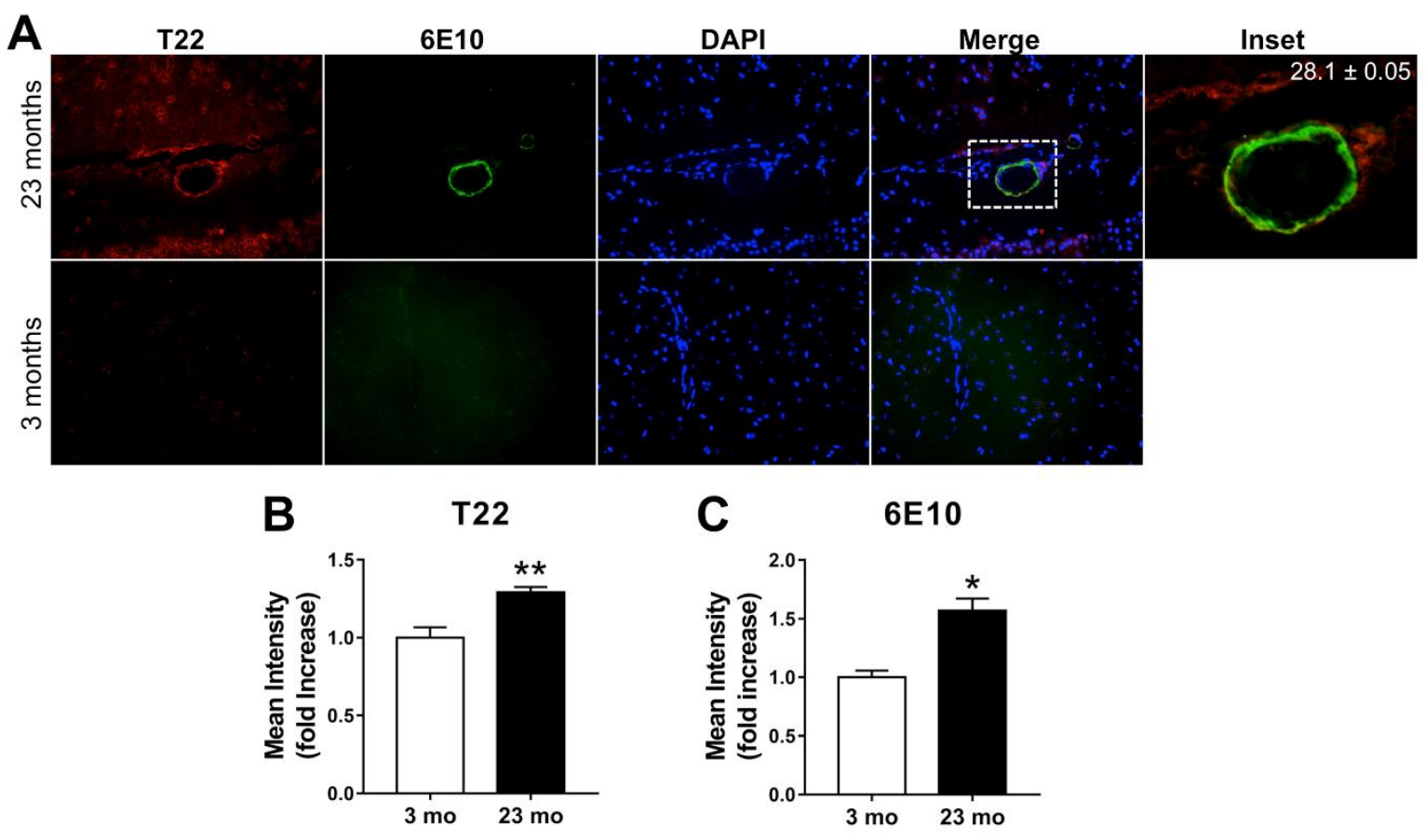

Figure 4. Age-dependent increase of oligomeric tau and fibrillar amyloid pathology in cerebrovasculature of Tg2576 mice. (A) Representative images of brain sections from 23-month-old (top) and 3-month-old (bottom) transgenic Tg2576 mice reacted with antibodies specific for tau oligomers (T22, red) and amyloid- $\beta$ (A $\beta, 6 \mathrm{E} 10$, green). Mander's colocalization coefficient suggests partial association of oligomeric tau and fibrillar $A \beta$ in cerebrovasculature of 23 -month-old mice. Because both T22 and $A \beta$ immunoreactivity were absent in 3-month-old mice, a colocalization coefficient could not be computed for this group. Mean percent colocalization \pm SEM are shown in the inset, which was merged without DAPI. (B) Quantitative analysis of T22-specific immunoreactivity shows increased cerebrovascular oligomeric tau deposition in 23 month-old compared to 3 month-old Tg2576 mice $(* *, \mathrm{t}(19)=3.87, p=0.010)$. (C) Quantitative analysis of $6 \mathrm{E} 10$-specific immunoreactivity shows an age-dependent increase in cerebrovascular $A \beta$ deposition in 23 month-old compared to 3 month-old mice $(*, t(20)=2.62, \mathrm{p}=0.016)$. $\mathrm{n}=3$; 10-15 sections from each sample were analyzed for tau oligomers. All sections were positive for tau oligomers. All of the transgenic Tg2576 mice used were positive for both Abeta and tau oligomers.

\section{DISCUSSION}

The major finding of the present study is that tau oligomers accumulate in cerebral microvasculature of human patients with AD and PSP, in association with vascular endothelial and smooth muscle cells. Cerebrovascular deposition of tau oligomers was also found in DLB patients, albeit to a lesser extent than in AD and PSP. Further, our studies show that tau oligomers accumulate in cerebral microvasculature of $\operatorname{Tg} 2576$ mice, a model that recapitulates CAA, at advanced stages of
AD-like progression and vascular histopathology, and that they partially associate with cerebrovascular $A \beta$ lesions.

These findings have important relevance for studies pertaining to the microvascular etiology of AD. Recent studies from our laboratory and others demonstrated tau oligomers constitute a distinct toxic species in $\mathrm{AD}[18,20$, $34,35]$. On the basis of previous in vitro findings showing that tau oligomers are cytotoxic at low nanomolar concentrations $[36,37]$ we predict that cerebral microvascular accumulation of tau oligomers impairs the 
function of endothelial cells and smooth muscle cells. Significant evidence exists that cerebral microvascular dysfunction contributes to the pathogenesis of $\mathrm{AD}$ [1-3]. Thus, future studies should determine how oligomeric tau impacts neurovascular coupling mechanisms, endothelial function and blood brain barrier integrity in $\mathrm{AD}$ and other tauopathies.

Cerebral microbleeds are small chronic brain hemorrhages [9-11] caused by structural weakening of the cerebral microvessels, which exacerbate brain damage and functional decline in $\mathrm{AD}$ patients. In theory, deposition of oligomeric tau in the cerebral microvessels may contribute to the pathogenesis of cerebral microbleeds by causing localized cell death and/or structural remodeling of the extracellular matrix. These possibilities should be tested in future studies.

Recently we demonstrated that specific removal of tau oligomers through passive immunotherapy using a tau oligomer-specific monoclonal antibody improves cognitive function without disrupting large NFTs in several tauopathy mouse models including the Tg2576, P301L, and Htau mice [38-41]. In light of our present findings further studies are warranted to determine whether immunotherapy using a tau oligomer-specific monoclonal antibody also removes tau oligomers from the cerebral microvessels and whether this improves/restores cerebral microvascular function. Initial evidence show that reducing tau levels rescues blood brain barrier integrity [42], suggesting that such experiments are worth undertaking.

The accumulation of $A \beta$ in cerebrovasculature is common in $\mathrm{AD}$ and can occur in the absence of specific $\mathrm{AD}$ changes both sporadically as well as in association with specific familial mutations in the APP gene [43]. The potential for adjacent deposition of oligomeric tau in cerebral microvessels [44] raises the possibility that tau aggravates microvascular $A \beta$ deposition and its consequences in $\mathrm{AD}$.

In conclusion, our findings add to the growing evidence for multifaceted microvascular involvement in the pathogenesis of $\mathrm{AD}$ and other neurodegenerative diseases. Accumulation of tau oligomers represents a potential novel mechanism by which functional and structural integrity of the cerebral microvessels may be compromised in dementias.

\section{Acknowledgements}

These studies were supported by the Mitchell Center for Neurodegenerative diseases (Kayed), the National Center for Advancing Translational Sciences, National Institutes of Health, through Grants AG054025 (Kayed), NS094557 (Kayed), as well as in part by Merit Review Award I01 BX002211-01A2 from the United States Department of
Veterans Affairs, Biomedical Laboratory Research and Development Service (Galvan) the San Antonio Medical Foundation (Galvan), a pilot award from the Institute for Integration of Medicine and Science UL1 TR001120 (Galvan), the JMR Barker Foundation, a William \& Ella Owens Medical Research Foundation Grant (Galvan), and the generous support from the Robert L. Bailey and daughter Lisa K. Bailey Alzheimer's Fund in memory of Jo Nell Bailey to VG. We also recognize the support of the Healthspan and Functional Assesment Core of the San Antonio Nathan Shock Center of Excellence in the Biology of Aging 2 P30 AG013319-21 (Galvan) and the Oklahoma Nathan Shock Center of Excellence in the Biology of Aging 3P30AG050911-02S1 to ZU, the National Institute on Aging R01-AG047879 and R01AG038747 (Ungvari). JBJ and CVS are supported by NIA Training Grant 2T32AG021890-11. The content is solely the responsibility of the authors and does not necessarily represent the official views of the NIH.

\section{References}

[1] Ruitenberg A, den Heijer T, Bakker SL, van Swieten JC, Koudstaal PJ, Hofman A, et al. (2005). Cerebral hypoperfusion and clinical onset of dementia: the Rotterdam Study. Ann Neurol, 57: 789-794

[2] Cantin S, Villien M, Moreaud O, Tropres I, Keignart S, Chipon E, et al. (2011). Impaired cerebral vasoreactivity to $\mathrm{CO} 2$ in Alzheimer's disease using BOLD fMRI. NeuroImage, 58: 579-587

[3] Iadecola C (2004). Neurovascular regulation in the normal brain and in Alzheimer's disease. Nat Rev Neurosci, 5: 347-360

[4] Zlokovic BV (2011). Neurovascular pathways to neurodegeneration in Alzheimer's disease and other disorders. Nat Rev Neurosci, 12: 723-738

[5] Corriveau RA, Bosetti F, Emr M, Gladman JT, Koenig JI, Moy CS, et al. (2016). The Science of Vascular Contributions to Cognitive Impairment and Dementia (VCID): A Framework for Advancing Research Priorities in the Cerebrovascular Biology of Cognitive Decline. Cell Mol Neurobiol, 36: 281-288

[6] Gorelick PB, Scuteri A, Black SE, Decarli C, Greenberg SM, Iadecola C, et al. (2011). Vascular contributions to cognitive impairment and dementia: a statement for healthcare professionals from the american heart association/american stroke association. Stroke, 42: 2672-2713

[7] Snyder HM, Corriveau RA, Craft S, Faber JE, Greenberg SM, Knopman D, et al. (2015). Vascular contributions to cognitive impairment and dementia including Alzheimer's disease. Alzheimers Dement, 11: 710-717

[8] Iturria-Medina Y, Sotero RC, Toussaint PJ, MateosPerez JM, Evans AC, Alzheimer's Disease Neuroimaging I (2016). Early role of vascular dysregulation on late-onset Alzheimer's disease based on 
multifactorial data-driven analysis. Nat Commun, 7: 11934

[9] Akoudad S, Gurol ME, Fotiadis P, Koudstaal PJ, Hofman A, Ikram MA, et al. (2016). Cerebral Microbleeds and Cerebrovascular Reactivity in the General Population: The EDAN Study. J Alzheimers Dis, 53: 497-503

[10] Park JH, Seo SW, Kim C, Kim GH, Noh HJ, Kim ST, et al. (2013). Pathogenesis of cerebral microbleeds: In vivo imaging of amyloid and subcortical ischemic small vessel disease in 226 individuals with cognitive impairment. Ann Neurol, 73: 584-593

[11] Zhao L, Arbel-Ornath M, Wang X, Betensky RA, Greenberg SM, Frosch MP, et al. (2015). Matrix metalloproteinase 9-mediated intracerebral hemorrhage induced by cerebral amyloid angiopathy. Neurobiol Aging, 36: 2963-2971

[12] Lin AL, Zheng W, Halloran JJ, Burbank RR, Hussong SA, Hart MJ, et al. (2013). Chronic rapamycin restores brain vascular integrity and function through $\mathrm{NO}$ synthase activation and improves memory in symptomatic mice modeling Alzheimer's disease. J Cereb Blood Flow Metab, 33: 1412-1421

[13] Miller EC, Teravskis PJ, Dummer BW, Zhao X, Huganir RL, Liao D (2014). Tau phosphorylation and tau mislocalization mediate soluble Abeta oligomer-induced AMPA glutamate receptor signaling deficits. Eur $\mathrm{J}$ Neurosci, 39: 1214-1224

[14] Rapoport M, Dawson HN, Binder LI, Vitek MP, Ferreira A (2002). Tau is essential to beta -amyloid-induced neurotoxicity. Proc Natl Acad Sci U S A, 99: 6364-6369

[15] Roberson ED, Scearce-Levie K, Palop JJ, Yan F, Cheng $\mathrm{IH}, \mathrm{Wu} \mathrm{T}$, et al. (2007). Reducing endogenous tau ameliorates amyloid beta-induced deficits in an Alzheimer's disease mouse model. Science, 316: 750754

[16] Grundke-Iqbal I, Iqbal K, Tung YC, Quinlan M, Wisniewski HM, Binder LI (1986). Abnormal phosphorylation of the microtubule-associated protein tau (tau) in Alzheimer cytoskeletal pathology. Proc Natl Acad Sci U S A, 83: 4913-4917

[17] Maeda S, Sahara N, Saito Y, Murayama M, Yoshiike Y, Kim H, et al. (2007). Granular tau oligomers as intermediates of tau filaments. Biochemistry, 46: 38563861

[18] Lasagna-Reeves CA, Castillo-Carranza DL, Sengupta U, Sarmiento J, Troncoso J, Jackson GR, et al. (2012). Identification of oligomers at early stages of tau aggregation in Alzheimer's disease. FASEB J, 5: 19461959

[19] Lasagna-Reeves CA, Castillo-Carranza DL, Sengupta U, Guerrero-Munoz MJ, Kiritoshi T, Neugebauer V, et al. (2012). Alzheimer brain-derived tau oligomers propagate pathology from endogenous tau. Scientific reports, 2: 700

[20] Lasagna-Reeves CA, Castillo-Carranza DL, Sengupta U, Clos AL, Jackson GR, Kayed R (2011). Tau oligomers impair memory and induce synaptic and mitochondrial dysfunction in wild-type mice. Mol Neurodegener, 6: 39
[21] Wu JW, Hussaini SA, Bastille IM, Rodriguez GA, Mrejeru A, Rilett K, et al. (2016). Neuronal activity enhances tau propagation and tau pathology in vivo. Nat Neurosci, 19: 1085-1092

[22] Gerson JE, Kayed R (2013). Formation and propagation of tau oligomeric seeds. Front Neurol, 4: 93

[23] Gerson JE, Sengupta U, Lasagna-Reeves CA, GuerreroMunoz MJ, Troncoso J, Kayed R (2014). Characterization of tau oligomeric seeds in progressive supranuclear palsy. Acta Neuropathol Commun, 2: 73

[24] Sengupta U, Guerrero-Munoz MJ, Castillo-Carranza DL, Lasagna-Reeves CA, Gerson JE, PaulucciHolthauzen AA, et al. (2015). Pathological interface between oligomeric alpha-synuclein and tau in synucleinopathies. Biol Psychiatry, 78: 672-683

[25] Vuono R, Winder-Rhodes S, de Silva R, Cisbani G, Drouin-Ouellet J, Network RIotEHsD, et al. (2015). The role of tau in the pathological process and clinical expression of Huntington's disease. Brain, 138: 19071918

[26] Hsiao K, Chapman P, Nilsen S, Eckman C, Harigaya Y, Younkin S, et al. (1996). Correlative memory deficits, Abeta elevation, and amyloid plaques in transgenic mice. Science, 274: 99-102

[27] Bergers G, Song S (2005). The role of pericytes in bloodvessel formation and maintenance. Neuro-oncology, 7: 452-464

[28] Fossati S, Ghiso J, Rostagno A (2012). Insights into caspase-mediated apoptotic pathways induced by amyloid-beta in cerebral microvascular endothelial cells. Neurodegener Dis, 10: 324-328

[29] Fossati S, Cam J, Meyerson J, Mezhericher E, Romero IA, Couraud PO, et al. (2010). Differential activation of mitochondrial apoptotic pathways by vasculotropic amyloid-beta variants in cells composing the cerebral vessel walls. FASEB J, 24: 229-241

[30] Saito S, Ihara M (2014). New therapeutic approaches for Alzheimer's disease and cerebral amyloid angiopathy. Front Aging Neurosci, 6: 290

[31] Irizarry MC, McNamara M, Fedorchak K, Hsiao K, Hyman BT (1997). APPSw transgenic mice develop agerelated A beta deposits and neuropil abnormalities, but no neuronal loss in CA1. J Neuropathol Exp Neurol, 56: 965-973

[32] Lanz TA, Carter DB, Merchant KM (2003). Dendritic spine loss in the hippocampus of young PDAPP and Tg2576 mice and its prevention by the ApoE2 genotype. Neurobiol Dis, 13: 246-253

[33] Lesne S, Koh MT, Kotilinek L, Kayed R, Glabe CG, Yang A, et al. (2006). A specific amyloid-beta protein assembly in the brain impairs memory. Nature, 440: 352357

[34] Lasagna-Reeves CA, Castillo-Carranza DL, GuerreroMuñoz MJ, Jackson GR, Kayed R (2010). Preparation and Characterization of Neurotoxic Tau Oligomers. Biochemistry, 49: 10039-10041

[35] Lasagna-Reeves CA, Castillo-Carranza DL, Jackson GR, Kayed R (2011). Tau oligomers as potential targets for immunotherapy for Alzheimer's disease and tauopathies. Current Alzheimer research, 8: 659-665 
[36] Tian H, Davidowitz E, Lopez P, Emadi S, Moe J, Sierks M (2013). Trimeric tau is toxic to human neuronal cells at low nanomolar concentrations. Int J Cell Biol, 2013: 260787

[37] Flach K, Hilbrich I, Schiffmann A, Gartner U, Kruger M, Leonhardt M, et al. (2012). Tau oligomers impair artificial membrane integrity and cellular viability. J Biol Chem, 287: 43223-43233

[38] Guerrero-Munoz MJ, Castillo-Carranza DL, Krishnamurthy S, Paulucci-Holthauzen AA, Sengupta U, Lasagna-Reeves CA, et al. (2014). Amyloid-beta oligomers as a template for secondary amyloidosis in Alzheimer's disease. Neurobiology of disease, 71: 14-23

[39] Castillo-Carranza DL, Sengupta U, Guerrero-Munoz MJ, Lasagna-Reeves CA, Gerson JE, Singh G, et al. (2014). Passive immunization with Tau oligomer monoclonal antibody reverses tauopathy phenotypes without affecting hyperphosphorylated neurofibrillary tangles. The Journal of neuroscience, 34: 4260-4272

[40] Gerson JE, Castillo-Carranza DL, Kayed R (2014). Advances in therapeutics for neurodegenerative tauopathies: moving toward the specific targeting of the most toxic tau species. ACS chemical neuroscience, 5: 752-769

[41] Castillo-Carranza DL, Gerson JE, Sengupta U, GuerreroMunoz MJ, Lasagna-Reeves CA, Kayed R (2014). Specific targeting of tau oligomers in Htau mice prevents cognitive impairment and tau toxicity following injection with brain-derived tau oligomeric seeds. J Alzheimers Dis, 40 Suppl 1: S97-S111

[42] Blair LJ, Frauen HD, Zhang B, Nordhues BA, Bijan S, Lin YC, et al. (2015). Tau depletion prevents progressive blood-brain barrier damage in a mouse model of tauopathy. Acta neuropathologica communications, 3: 8

[43] Attems J, Jellinger K, Thal DR, Van Nostrand W (2011). Review: sporadic cerebral amyloid angiopathy. Neuropathol Appl Neurobiol, 37: 75-93

[44] Williams S, Chalmers K, Wilcock GK, Love S (2005). Relationship of neurofibrillary pathology to cerebral amyloid angiopathy in Alzheimer's disease. Neuropathol Appl Neurobiol, 31: 414-421 